\title{
Stability of Sampled-Data Piecewise-Affine Systems Under State Feedback ${ }^{\star}$
}

\author{
Luis Rodrigues
}

Dept. of Mechanical and Industrial Engineering, Concordia University, 1515 St. Catherine Street, EV4.243
Montréal, QC H3G 2W1, Canada

\begin{abstract}
This paper addresses stability of sampled-data piecewise-affine (PWA) systems consisting of a continuous-time plant and a discrete-time emulation of a continuous-time state feedback controller. The paper presents conditions under which the trajectories of the sampled-data closed-loop system will exponentially converge to a neighborhood of the origin. Moreover, the size of this neighborhood will be related to bounds on perturbation parameters related to the sampling procedure, in particular, related to the sampling period. Finally, it will be shown that when the sampling period converges to zero the performance of the stabilizing continuous-time PWA state feedback controller can be recovered by the emulated controller.
\end{abstract}

Key words: Piecewise-Affine Systems, Switched Systems, Sampled-data Systems, Stability Analysis, Linear Matrix Inequalities.

\section{Introduction}

PWA systems are multi-model systems that offer a good modeling framework for complex dynamical systems involving nonlinear phenomena. State and output feedback control of continuous-time PWA systems have received increasing interest over the last years. The research work has concentrated on Lyapunov-based controller synthesis methods for continuous-time PWA systems Hassibi \& Boyd (1998); Johansson (2003); Johansson \& Rantzer (2000); Rodrigues \& How (2003); Rodrigues \& Boyd (2005). However, none of these approaches would be applicable directly to controller synthesis for computer-controlled or sampled-data PWA systems. This is the scenario mostly encountered in applications given the flexibility of control implementation in a microprocessor. References Hassibi \& Boyd (1998); Johansson (2003); Johansson \& Rantzer (2000); Rodrigues \& How (2003); Rodrigues \& Boyd (2005) consider continuous-time processes controlled by continuous-time controllers while the implementation in a microprocessor requires emulation of a continuous-

* This paper was not presented at any IFAC meeting. Corresponding author L. Rodrigues. Tel. 514-8482424. Fax 5148483175.

Email address: luisrod@encs.concordia.ca (Luis Rodrigues).

Preprint submitted to Automatica time controller as a discrete-time controller. Although linear sampled-data systems are a well-studied matter Chen \& Francis (1995), controller emulation for systems with possible discontinuities at the switching, such as sampled-data PWA systems, has not had many research contributions. In fact, only recently these systems have started to be addressed in the literature in references such as Imura (2003a,b); Azuma \& Imura (2004); Sun \& Ge (2002); Sun (2004); G. Zhai \& Yasuda (2004). The approach by Sun \& Ge (2002) established that, under certain conditions, the controllable subspaces of a continuous-time switched linear system and its discrete-time counterpart are the same. Canonical forms of switched linear systems based on controllability are presented in the more recent work of Sun (2004). The approach by G. Zhai \& Yasuda (2004) considers stability analysis of switched systems that can switch between a set of continuous-time plants and a set of discretetime plants but does not handle sampled-data systems involving a cascade of a discrete-time system between a sample-and-hold and a continuous-time system. Furthermore, it does not address controller design. The approach by Imura (2003a,b); Azuma \& Imura (2004) was probably the first where the term "sampled-data PWA systems" is used, although the systems described in this work do not posses the typical structure of a continuoustime plant being controlled by a discrete-time controller. The problem addressed in Imura (2003a,b); Azuma \& 
Imura (2004) is one where the controller is continuoustime and the switching events are the ones controlled by the system logic inside a computer. In other words, in these systems it is assumed that the designer has command over the switching times of the system. The preliminary study of Imura $(2003 \mathrm{a}, \mathrm{b})$ is interesting as it highlights important limitations of current discretetime PWA control methodologies when applied to the control of a physical continuous-time system. As mentioned in Imura (2003a) unexpected phenomena such as chattering can occur, depending on the switching times. This increases the interest in studying computer implementations of controllers designed in continuous-time.

This paper addresses the classical structure of a sampleddata system whereby the system is continuous-time and the controller is being implemented (emulated) in discrete-time inside a computer. Previous approaches to this classical structure can be classified into two categories: i) discrete-time controller design to a discretetime approximation of the continuous-time plant and ii) continuous-time controller design to a continuoustime plant followed by discrete-time emulation of the controller. To the best of the author's knowledge the only previous work in sampled-data PWA systems is the work of Imura et. al. Imura (2003a,b); Azuma \& Imura (2004) which, as already stated, does not address the classical structure of interest in this paper. For papers in sampled-data control for nonlinear systems that fall into category i) we refer the reader to Nesic \& Laila (2002) and references therein. For papers in sampleddata control for nonlinear systems that fall under category ii) we refer the reader to Khalil (2004) and references therein. Note that these papers always assume the plant dynamics to be locally Lipschitz. Therefore they do not include the possibility of having PWA dynamics that are switched with possible discontinuities in the plant dynamics at the switching. The interesting paper by Nesic \& Teel (2004) also falls under category i) described above but offers the advantage of treating the plant model as a differential inclusion, thus possibly enabling discontinuous vector fields. In fact, one of the examples described in Nesic \& Teel (2004) deals with a hysteresis switched controller. Although potentially applicable to PWA systems, Nesic \& Teel (2004) does not address the problem of interest here, namely stability and performance recovery by emulation of a continuous-time PWA controller. Furthermore, in the framework of Nesic \& Teel (2004), the plant dynamics must be embedded in a differential inclusion, which can potentially lead to conservative results instead of handling the PWA dynamics directly.

The paper starts by stating the problem assumptions. Then, the stability of the sampled-data system when a continuous-time controller is emulated in discrete-time is analyzed. A numerical example is included to show an application of the main stability result. Finally, the paper closes by stating the conclusions.

\section{Problem Assumptions}

It is assumed that a PWA system and a corresponding partition of the state space with polytopic cells $\mathcal{R}_{i}, i \in$ $\mathcal{I}=\{1, \ldots, M\}$ are given (see Rodrigues \& How (2001) for generating such a partition). Following Johansson (2003); Hassibi \& Boyd (1998), each cell is constructed as the intersection of a finite number $\left(p_{i}\right)$ of half spaces

$$
\mathcal{R}_{i}=\left\{z \in \mathbb{R}^{n} \mid H_{i}^{T} z-g_{i}<0\right\},
$$

where $H_{i}=\left[h_{i 1} \ldots h_{i p_{i}}\right] \in \mathbb{R}^{n \times p_{i}}, g_{i}=\left[g_{i 1} \ldots g_{i p_{i}}\right]^{T} \in$ $\mathbb{R}^{p_{i}}$. Moreover the sets $\mathcal{R}_{i}$ partition a subset of the state space $\mathcal{X} \subset \mathbb{R}^{n}$ such that $\cup_{i=1}^{M} \overline{\mathcal{R}}_{i}=\mathcal{X}, \mathcal{R}_{i} \cap \mathcal{R}_{j}=\emptyset, i \neq$ $j$, where $\overline{\mathcal{R}}_{i}$ denotes the closure of $\mathcal{R}_{i}$. Within each cell the dynamics are affine of the form

$$
\dot{z}(t)=A_{i} z(t)+b_{i}+B_{i} u(t),
$$

where $z(t) \in \mathbb{R}^{n}, u(t) \in \mathbb{R}^{m}$ and $b_{i} \in \mathbb{R}^{n}$. For system (2), we adopt the following definition of solutions.

Definition 1 Johansson (2003) Let $z(t) \in \mathcal{X}$ be an $a b$ solutely continuous function. Then $z(t)$ is a trajectory of the system (2) on $\left[t_{0}, t_{f}\right]$ if, for almost all $t \in\left[t_{0}, t_{f}\right]$ and Lebesgue measurable $u(t)$, the equation $\dot{z}(t)=A_{i} z(t)+$ $b_{i}+B_{i} u(t)$ holds for all $i$ such that $z(t) \in \overline{\mathcal{R}}_{i}$.

Any two cells sharing a common facet will be called level-1 neighboring cells. Let $\mathcal{N}_{i}=\{$ level- 1 neighboring cells of $\mathcal{R}_{i}$. Vectors $c_{i j} \in \mathbb{R}^{n}$ and scalars $d_{i j}$ will then exist such that the facet boundary between cells $\mathcal{R}_{i}$ and $\mathcal{R}_{j}$ is contained in the hyperplane described by $\left\{z \in \mathbb{R}^{n} \mid c_{i j}^{T} z-d_{i j}=0\right\}$, for $i=1, \ldots, M, j \in \mathcal{N}_{i}$. A parametric description of the boundaries is

$$
\overline{\mathcal{R}_{i}} \cap \overline{\mathcal{R}_{j}} \subseteq\left\{z=l_{i j}+F_{i j} s \mid s \in \mathbb{R}^{n-1}\right\}
$$

for $i=1, \ldots, M, j \in \mathcal{N}_{i}$, where $F_{i j} \in \mathbb{R}^{n \times(n-1)}$ (full rank) is the matrix whose columns span the null space of $c_{i j}^{T}$ and $l_{i j} \in \mathbb{R}^{n}$ is given by $l_{i j}=c_{i j}\left(c_{i j}^{T} c_{i j}\right)^{-1} d_{i j}$. It is further assumed that matrices $E_{i}$ and $f_{i}$ exist such that $\mathcal{R}_{i} \subseteq \varepsilon_{i}$,

$$
\varepsilon_{i}=\left\{z \mid\left\|E_{i} z+f_{i}\right\| \leq 1\right\} .
$$

This ellipsoidal covering is especially useful in the case where $\mathcal{R}_{i}$ is a slab because in this case the matrices $E_{i}$ and $f_{i}$ are guaranteed to exist and the covering (having one degenerate ellipsoid $\varepsilon_{i}$ ) is exact, i.e., $\varepsilon_{i} \subseteq \mathcal{R}_{i}$ and $\mathcal{R}_{i} \subseteq \varepsilon_{i}$. More precisely, if $\mathcal{R}_{i}=\left\{z \mid d_{1}<c_{i}^{\bar{T}} z<\right.$ $\left.d_{2}\right\}$, then the degenerate ellipsoid is described by $E_{i}=$ $2 c_{i}^{T} /\left(d_{2}-d_{1}\right)$ and $f_{i}=-\left(d_{2}+d_{1}\right) /\left(d_{2}-d_{1}\right)$. Finally it is assumed, without loss of generality, that the control objective is to stabilize the system to the origin. 


\section{Stability of Sampled-Data PWA Systems}

In this section a stability result is presented for the closed-loop sampled-data system that is obtained when a continuous-time state feedback controller is implemented on a digital computer. It is assumed that a continuous-time state feedback controller parameterized by $K_{i} \in \mathbb{R}^{m \times n}$ and $m_{i} \in \mathbb{R}^{m}$ in the form

$$
u=K_{i} z+m_{i}, \quad z \in \mathcal{R}_{i}
$$

has already been designed such that the continuous-time closed-loop system is exponentially stable. ${ }^{1}$ It is also assumed that the state $z$ of the system is measured at a sampling rate $f_{s}=T^{-1}, T>0$, and that the controller in the feedback loop appears between a sampler and a zero-order-hold. At the sampling instants, the plant state and the sampled state overlap and therefore the sampled-data system is described by

$$
\dot{z}=A_{j} z+b_{j}+B_{j} K_{j} z(k T)+B_{j} m_{j},
$$

for $z(t) \in \mathcal{R}_{j}, z(k T) \in \mathcal{R}_{j}$. However, for a given time $t$ that is not a sampling instant, the general situation that should be considered is the one in which the state of the plant is in region $\mathcal{R}_{i}$ and the most recently sampled state is in region $\mathcal{R}_{j}$ with possibly $i \neq j$. The system is then described by the differential equation

$$
\dot{z}=A_{i} z+b_{i}+B_{i} K_{j} z(k T)+B_{i} m_{j},
$$

for $z(t) \in \mathcal{R}_{i}, z(k T) \in \mathcal{R}_{j}$. This equation can be rewritten in the perturbed form

$$
\dot{z}=\bar{A}_{i} z+\bar{b}_{i}+B_{i} \delta_{i j}
$$

for $z(t) \in \mathcal{R}_{i}, z(k T) \in \mathcal{R}_{j}$, where $\bar{A}_{i}=A_{i}+B_{i} K_{i}, \bar{b}_{i}=$ $b_{i}+B_{i} m_{i}$ and

$\delta_{i j}=K_{j}(z(k T)-z(t))+\left(K_{j}-K_{i}\right) z(t)+\left(m_{j}-m_{i}\right)$.

Note that the first term in (9) represents the perturbation due to the error between the last available sample of the state and its current value. The second and third terms are associated with the perturbation due to the state and its most recent sample being possibly in different regions. The second term represents the perturbation due to a difference in the gain matrices in regions $\mathcal{R}_{i}$ and $\mathcal{R}_{j}$ and the third term represents the perturbation due to a difference in the affine control terms. Given a continuous-time controller of the form (5), the first step in the procedure outlined in this paper is to search for a quadratic Lyapunov function of the form

$$
V(z)=z^{T} P z
$$

\footnotetext{
1 For optimization programs whose solution (when it exists) yields exponentially stabilizing PWA controllers see Rodrigues \& How (2003); Rodrigues \& Boyd (2005).
}

that proves stability of the continuous-time closed-loop system. This can be done by solving for fixed $\alpha \geq 0$ the following set of LMIs (see for example Rodrigues \& Boyd (2005) for details on the derivation of these conditions):

$$
\begin{array}{r}
P=P^{T}>0, \lambda_{i}<0, i=1, \ldots, M \\
{\left[\begin{array}{rr}
\bar{A}_{i}^{T} P+P \bar{A}_{i}+\alpha P+\lambda_{i} E_{i}^{T} E_{i} P \bar{b}_{i}+\lambda_{i} E_{i}^{T} f_{i} \\
\left(P \bar{b}_{i}+\lambda_{i} E_{i}^{T} f_{i}\right)^{T} & -\lambda_{i}\left(1-f_{i}^{T} f_{i}\right)
\end{array}\right]<0}
\end{array}
$$

The results that follow assume that such a Lyapunov function can be found. Note however that not all continuous-time PWA systems that are stable admit a globally quadratic Lyapunov function (see Johansson (2003) for counter-examples).

\subsection{Conditions Independent of the Sampling Period}

We now present the first result of this section. It gives conditions under which the trajectories of the sampleddata system (8) converge to a region around the closedloop equilibrium point. Furthermore, it relates the size of this region to a measure of the perturbation term in the closed-loop system. In what follows, unless otherwise indicated, the time dependence of the indices $i, j$ will be omitted for simplicity.

Theorem 2 Assume a Lyapunov function of the form (10) is found and is defined in $\mathcal{X} \subseteq \mathbb{R}^{n}$. Let the condition number of $P$ be $\chi_{P}=\frac{\sigma_{\max }(P)}{\sigma_{\min }(P)}$. Assume there are finite constants $N_{i j}>0, \Delta_{K_{i j}} \geq 0$, such that $\left\|\delta_{i j}\right\| \leq N_{i j}+\Delta_{K_{i j}}\|z\|, i, j=1, \ldots, M$. Let $N=$ $\max _{i, j=1, \ldots, M}\left(N_{i j}\right), \Delta_{K}=\max _{i, j=1, \ldots, M}\left(\Delta_{K_{i j}}\right), B=$ $\max _{i=1, \ldots, M}\left\|B_{i}\right\|$. Define

$$
\mu_{\theta}=\frac{2 \chi_{P} B}{\alpha \theta-2 \chi_{P} B \Delta_{K}} N
$$

and the region

$$
\mathcal{S}_{\theta}=\left\{z \in \mathcal{X} \mid\|z\| \leq \mu_{\theta}\right\}
$$

for any positive constant $\theta<1$ that verifies

$$
\Delta_{K}<\frac{\alpha \theta}{2 \chi_{P} B}
$$

Then, if (12) is verified, the trajectories of the closedloop sampled-data system (8) converge exponentially to the set

$$
\Omega=\left\{z \in \mathcal{X} \mid V(z) \leq \sigma_{\max }(P) \mu_{\theta}^{2}\right\}
$$

Proof: For $z(t) \in \mathcal{R}_{i}, z(k T) \in \mathcal{R}_{j}$, using the dynamics $(8)$, the derivative of the candidate Lyapunov function 
(10) along the trajectories of the system is

$$
\dot{V}(z)=\left[\begin{array}{c}
z \\
1
\end{array}\right]^{T}\left[\begin{array}{cc}
\bar{A}_{i}^{T} P+P \bar{A}_{i} & P \bar{b}_{i} \\
\left(P \bar{b}_{i}\right)^{T} & 0
\end{array}\right]\left[\begin{array}{l}
z \\
1
\end{array}\right]+2 z^{T} P B_{i} \delta_{i j}
$$

However, note that if a quadratic Lyapunov function is found by solving (11), using the $\mathcal{S}$-procedure (see Rodrigues \& Boyd (2005) for details) it can be shown that for $z \in \mathcal{R}_{i}$

$$
\left[\begin{array}{c}
z \\
1
\end{array}\right]^{T}\left[\begin{array}{cc}
\bar{A}_{i}^{T} P+P \bar{A}_{i} P \bar{b}_{i} \\
\left(P \bar{b}_{i}\right)^{T} & 0
\end{array}\right]\left[\begin{array}{c}
z \\
1
\end{array}\right]<-\alpha z^{T} P z .
$$

Therefore, for $z \in \mathcal{R}_{i}, z(k T) \in \mathcal{R}_{j}$ it follows that

$$
\dot{V}(z)<-\alpha z^{T} P z+2 z^{T} P B_{i} \delta_{i j}
$$

Taking norms and using the bounds

$$
\left\|\delta_{i j}\right\| \leq N_{i j}+\Delta_{K_{i j}}\|z\| \leq N+\Delta_{K}\|z\|
$$

and $-z^{T} P z \leq-\sigma_{\min }(P)\|z\|^{2}$ yields

$\dot{V}(z)<-\alpha \sigma_{\min }(P)\|z\|^{2}+2\|z\| \sigma_{\max }(P) B\left(N+\Delta_{K}\|z\|\right)$

or, for any positive constant $\theta<1$

$$
\begin{array}{r}
\dot{V}(z)<-(1-\theta) \alpha \sigma_{\min }(P)\|z\|^{2}- \\
\theta \alpha \sigma_{\min }(P)\|z\|^{2}+2\|z\| \sigma_{\max }(P) B\left(N+\Delta_{K}\|z\|\right) .
\end{array}
$$

Therefore, for $0<\theta<1$, we have

$$
\dot{V}(z)<-(1-\theta) \alpha \sigma_{\min }(P)\|z\|^{2} \leq-(1-\theta) \chi_{P}^{-1} \alpha V(z)
$$

for

$$
\|z\|>\frac{2 \chi_{P} B}{\alpha \theta-2 \chi_{P} B \Delta_{K}} N
$$

provided

$$
\Delta_{K}<\frac{\alpha \theta}{2 \chi_{P} B} .
$$

Note that although condition (13) is only valid inside each region of the partition of the state space, it also guarantees that no unstable sliding modes can be generated at the boundaries because the Lyapunov function is of class $\mathcal{C}^{1}$ (see Samadi \& Rodrigues (2007) for more details). As a result of (13), for $z \in \mathbb{R}^{n} \backslash \mathcal{S}_{\theta}$,

$$
V(z(t))<V\left(z\left(t_{0}\right)\right) e^{-(1-\theta) \chi_{P}^{-1} \alpha\left(t-t_{0}\right)}
$$

Using the relation $\sigma_{\min }(P)\|z\|^{2} \leq V(z) \leq \sigma_{\max }(P)\|z\|^{2}$ we can conclude that for $z \in \mathbb{R}^{n} \backslash \mathcal{S}_{\theta}$,

$$
\|z(t)\| \leq\left\|z\left(t_{0}\right)\right\| \chi_{P}^{\frac{1}{2}} e^{-0.5(1-\theta) \chi_{P}^{-1} \alpha\left(t-t_{0}\right)}
$$

Thus, there will be a positive and finite time $t_{1}^{\theta}$ such that $z\left(t_{1}^{\theta}\right) \in \mathcal{S}_{\theta}$ for any positive constant $\theta<1$ that verifies (14). Note that $\mathcal{S}_{\theta} \subseteq \Omega$. This can be proved by contradiction. Assume that it is not true that $\mathcal{S}_{\theta} \subseteq$ $\Omega$. Then, there exists at least one $z_{0} \in \mathcal{S}_{\theta}$ for which $z_{0}^{T} P z_{0}>\sigma_{\max }(P) \mu_{\theta}^{2}$, a contradiction. Since $\dot{V} \leq 0$ at the boundary of $\Omega, \Omega$ is an invariant set for system (8). Consequently, since $z\left(t_{1}^{\theta}\right) \in \mathcal{S}_{\theta} \subseteq \Omega, z(t) \in \Omega$ for all $t \geq t_{1}^{\theta}$ and for all $0<\theta<1$ that verifies (14).

Remark 3 This result relates the size of the region to which the trajectories converge to the size of the perturbations. The size of the region decreases with the size of the perturbations, as expected. Note that for the case where $K_{i}=K_{j}, \Delta_{K}=0$ and (14) is automatically verified.

Remark 4 Bounds on $\delta_{i j}$ can be easily obtained in the case where all polytopic regions are bounded, by noticing that $\|z(k T)-z(t)\| \leq \max _{x \in \mathcal{R}_{i}, y \in \mathcal{R}_{j}}\|x-y\|$. These bounds are however potentially conservative and better ways of obtaining them should be investigated. In particular, the bound should depend on the sampling period $T$.

The next section relates the bound on $\|z(k T)-z(t)\|$ to the sampling period $T$ and offers a less conservative result that enables us to prove that if the sampling period converges to zero then the system is practically exponentially stable to the origin and the continuous-time behavior is recovered.

\subsection{Conditions Dependent of the Sampling Period}

Integrating equation (7) for $t \in[k T,(k+1) T]$ yields

$$
\begin{array}{r}
z(t)-z(k T)=\int_{k T}^{t} A_{i(\tau)} z(\tau) d \tau+\int_{k T}^{t} b_{i(\tau)} d \tau+ \\
\int_{k T}^{t} B_{i(\tau)} d \tau\left(K_{j} z(k T)+m_{j}\right)
\end{array}
$$

Thus, letting $A=\max _{i=1, \ldots, M}\left\|A_{i}\right\|, b=\max _{i=1, \ldots, M}\left\|b_{i}\right\|$, $B=\max _{i=1, \ldots, M}\left\|B_{i}\right\|$ yields

$$
\begin{array}{r}
\|z(t)-z(k T)\| \leq A \int_{k T}^{t}\|z(\tau)\| d \tau+ \\
\quad(t-k T)\left(b+B\left\|K_{j} z(k T)+m_{j}\right\|\right)
\end{array}
$$

Since all possible dynamics in a PWA system with coefficients independent of the partition are affine, finite escape times cannot occur and therefore there will be a finite constant $Z(k, T)=\sup _{k T \leq t \leq k T+T}\|z(t)\|$ such that

$$
\|z(t)\|_{k T \leq t \leq k T+T} \leq Z(k, T)
$$


Using the bound (17) in expression (16) leads to

$$
\begin{array}{r}
\|z(t)-z(k T)\| \leq(t-k T)(A Z(k, T)+b+ \\
\left.B\left\|K_{j} z(k T)+m_{j}\right\|\right)
\end{array}
$$

Remark 5 Note that the bound $Z(k, T)$ gets smaller as the sampling time decreases and when $T \rightarrow 0, Z(k, T) \rightarrow$ $\|z(k T)\|$. Note further that the Euler approximation for integration would lead to $Z(k, T)=\|z(k T)\|$ because $\int_{k T}^{t}\|z(\tau)\| d \tau \simeq\|z(k T)\|(t-k T)$.

Letting $K=\max _{i=1, \ldots, M}\left\|K_{i}\right\|, m=\max _{i=1, \ldots, M}\left\|m_{i}\right\|$,

$$
\begin{array}{r}
\|z(t)-z(k T)\| \leq(t-k T)(A Z(k, T)+b+ \\
B K\|z(k T)\|+B m)
\end{array}
$$

The worst possible (highest) bound is the one corresponding to $t=(k+1) T$, which leads to

$\|z(t)-z(k T)\| \leq T(A Z(k, T)+b+B K\|z(k T)\|+B m)$

Recall that the expression for the perturbations developed in (9) was

$\delta_{i j}=K_{j}(z(k T)-z(t))+\left(K_{j}-K_{i}\right) z(t)+\left(m_{j}-m_{i}\right)$.

Let now $\Delta_{K_{i j}}=\left\|K_{j}-K_{i}\right\|, \Delta_{m_{i j}}=\left\|m_{j}-m_{i}\right\|$. Then we can write

$$
\left\|\delta_{i j}\right\| \leq K\|z(t)-z(k T)\|+\Delta_{K_{i j}}\|z(t)\|+\Delta_{m_{i j}}
$$

and therefore using (17) and (20) this finally yields

$$
\left\|\delta_{i j}\right\| \leq N_{i j}(k, T)+\Delta_{K_{i j}}\|z\|, i, j=1, \ldots, M
$$

where

$$
N_{i j}(k, T)=\Delta_{m_{i j}}+K T(\bar{A} Z(k, T)+\bar{b})
$$

and $\bar{A}=A+B K, \bar{b}=b+B m$. Using this bound and Theorem 2 the following result can now be stated.

Corollary 6 Assume a Lyapunov function of the form (10) is found and is defined in $\mathcal{X} \subseteq \mathbb{R}^{n}$. Let the condition number of $P$ be $\chi_{P}=\frac{\sigma_{\max }(P)}{\sigma_{\min }(P)}$. Let $N_{i j}(k, T)$ be defined as in (24) where $Z(k, T)=\sup _{k T \leq t \leq k T+T}\|z(t)\|$. Define

$$
\bar{N}_{i j}(T)=\Delta_{m_{i j}}+K T(\bar{A} \bar{Z}(T)+\bar{b})
$$

where

$$
\bar{Z}(T)=\lim _{L \rightarrow \infty} \max _{k \in\{0, \ldots, L\}} Z(k, T)
$$

and

$$
\begin{array}{r}
A=\max _{i=1, \ldots, M}\left\|A_{i}\right\|, b=\max _{i=1, \ldots, M}\left\|b_{i}\right\|, \\
B=\max _{i=1, \ldots, M}\left\|B_{i}\right\|, \Delta_{K}=\max _{i, j=1, \ldots, M}\left\|K_{j}-K_{i}\right\| .
\end{array}
$$

Furthermore, let $N(T)=\max _{i, j=1, \ldots, M}\left(\bar{N}_{i j}\right)$. Define

$$
\mu_{\theta}(T)=\frac{2 \chi_{P} B}{\alpha \theta-2 \chi_{P} B \Delta_{K}} N(T)
$$

and the region

$$
\mathcal{S}_{\theta}(T)=\left\{z \in \mathcal{X} \mid\|z\| \leq \mu_{\theta}(T)\right\}
$$

for any positive constant $\theta<1$ that verifies

$$
\Delta_{K}<\frac{\alpha \theta}{2 \chi_{P} B}
$$

Then, in the absence of sliding modes, if (27) is verified it follows that:

(1) The trajectories of the closed-loop sampled-data system (8) converge exponentially to the set

$$
\Omega(T)=\left\{z \in \mathcal{X} \mid V(z) \leq \sigma_{\max }(P) \mu_{\theta}^{2}(T)\right\} .
$$

(2) When $T \rightarrow 0$, the trajectories of the closed-loop sampled-data system (8) are practically exponentially stable to the origin. By this it is meant that $z(t) \rightarrow 0$ exponentially a.e when $T \rightarrow 0$.

Proof: Result 1) follows directly from the proof of Theorem 2. Result 2) follows from the facts that:

In the absence of sliding modes, chattering phenomena is ruled out in closed-loop. Therefore, for any finite $t^{\star}$, there will be a finite number $N^{\star}\left(t^{\star}, T\right)$ of switchings in the time period $\left[0, t^{\star}\right]$.

At $t=k T$ the dynamics of the system will be governed by (6) where $j$ is the index of the region where the state lies at or immediately after $k T$. Notice that (6) is an affine differential equation with constant coefficients and finite escape times are not possible, so $z(t)$ is bounded. Notice also that until a switch occurs, the solution of $(6)$ is continuous and given by the variation of constants

$$
\begin{aligned}
z(t) & =\Phi_{j}(t-k T) z(k T)+\Gamma_{j}(t-k T) u_{j} \\
u_{j} & =b_{j}+B_{j}\left(K_{j} z(k T)+m_{j}\right)
\end{aligned}
$$

where $\Phi_{j}(t-k T)=e^{A_{j}(t-k T)}$ and $\Gamma_{j}(t-k T)=$ $\int_{0}^{t-k T} e^{A_{j} \tau} d \tau$. Note also that

$\|z(t)-z(k T)\| \leq\left\|\Phi_{j}+\Gamma_{j} B_{j} K_{j}-I\right\| \bar{Z}(T)+\left\|\Gamma_{j}\right\|\left\|\bar{b}_{j}\right\|$, 
and thus $\|z(t)-z(k T)\|$ is also bounded since $\bar{Z}(T)$ must stay bounded due to the impossibility of finite escape times for PWA systems with constant coefficients not dependent on the partition. Furthermore, the first switching outside of region $j$ will only occur when $w_{j i}(z)=$ $h_{j i}^{T} z-g_{j i}=0$ for some $i \in \mathcal{N}_{j}$. Since $z(t)$ and $w_{j i}(z)$ are continuous and $w_{j i}(z) \neq 0$ right after the update of the state at $t=k T, w_{j i}(z) \neq 0$ for at least a time interval with some positive measure $\epsilon_{i j} \leq T$ until the occurence of the next switch. Because of this fact, $N^{\star}\left(t^{\star}, T\right)$ cannot grow unbounded as $T \rightarrow 0$. Until the occurence of a switch, $\Delta_{m_{i j}}=0, \Delta_{K_{i j}}=0$.

From the previous point, we conclude that for any $T>$ $0, k \geq 0$ the Lebesgue measure of the set

$$
S_{k T}^{i, j}=\left\{t \in[k T,(k+1) T] \mid \Delta_{K_{i j}}(t), \Delta_{m_{i j}}(t)>0\right\}
$$

verifies

$$
\mu\left(S_{k T}^{i, j}\right) \leq T-\epsilon_{i j}(T)<T
$$

Thus, for any finite $t^{\star}$ the set $\mathcal{S}=\cup_{k: k T<t^{\star}} \mathcal{S}_{k T}^{i, j}$ will have bounded Lebesgue measure $\mu(\mathcal{S}) \leq N^{\star} T$. As $T \rightarrow 0$, the Lebesgue measure of this set will also converge to zero because $N^{\star}\left(t^{\star}, T\right)$ cannot grow unbounded as $T \rightarrow 0$.

Therefore $\Delta_{K_{i j}} \rightarrow 0, \Delta_{m_{i j}} \rightarrow 0$ as $T \rightarrow 0$ except possibly on a set of time instants that has Lebesgue measure converging to zero. Thus, the set of times $t$ for which $i(t), j(t)$ are different converges to zero almost everywhere as $T \rightarrow 0$ so $i=j$ a.e when $T \rightarrow 0$.

$Z(k, T) \rightarrow\|z(k T)\|$ when $T \rightarrow 0$ and, as seen before, $\Delta_{m_{i j}} \rightarrow 0$ a.e as $T \rightarrow 0$. This together with the fact that $\|z(k T)\|$ is bounded for any $k \geq 0$ implies by $(25)$ and (26) that $\bar{N}_{i j}(T) \rightarrow 0$ a.e when $T \rightarrow 0$. Thus $\mu_{\theta}(T) \rightarrow$ 0 a.e when $T \rightarrow 0$.

Following the rationale in the proof of Theorem 2, the previous points show that the closed-loop system trajectories converge exponentially to the set $\Omega(T)$ whose size converges to zero as $\mu_{\theta}(T) \rightarrow 0$ a.e when $T \rightarrow 0$. In fact, following the arguments of the proof of Theorem 2 , when $T \rightarrow 0$ the Lyapunov function $V(z)$ decreases exponentially except possibly for a set of times whose Lebesgue measure converges to zero as $T \rightarrow 0$. However, for this set of times, as discussed, $z(t)$ remains bounded and, by expression $(20), z(t)-z(k T)$ remains bounded and is small for small $T$. Since the Lyapunov function is of class $\mathcal{C}^{2}$ on $z$, the Lyapunov function also remains bounded. Given that the Lyapunov function remains bounded for these sets of time and these time instants form a set whose Lebesgue measure converges to zero as $T \rightarrow 0, V(t)<V\left(t_{0}\right) e^{-(1-\theta) \chi_{P}^{-1} \alpha\left(t-t_{0}\right)}$, a.e. and the result of the theorem is established by the fact that $\sigma_{\min }(P)\|z(t)\|^{2} \leq V(z) \leq \sigma_{\max }\|z(t)\|^{2}$.
Remark 7 This result formally establishes the very important and desired property that a sampled-data PWA system converges to a closed-loop continuous-time PWA system when the sampling period converges to zero. As desired, all the stability guarantees for the closed-loop continuous-time system can be recovered.

Remark 8 The result assumes the absence of sliding modes. Sliding modes can indeed be ruled out in feedback for PWA systems with hyperplane boundaries if the component of the vector fields perpendicular to the boundaries is continuous across the boundaries. This idea was first suggested for PWA systems in Rodrigues 85 How (2003) to avoid the generation of sliding modes in closedloop. If the feedback construction suggested in Rodrigues E How (2003) is used, it can be shown following the reasoning explained in Rodrigues 83 How (2003) that sliding modes are still ruled out in feedback for sampled-data $P W A$ systems if the additional constraints $B_{i}=B_{j}=$ $B, c_{i j}^{T} B\left(K_{j}-K_{i}+m_{j}-m_{i}\right)=0, \forall i=1, \ldots, M, \forall j \in$ $\mathcal{N}_{i}$ are verified. Notice that these constraints are linear in the controller parameters and can easily be included in the optimization procedure suggested in Rodrigues $\& 3$ How (2003) for systems with a constant input matrix B (such as the one presented in the example of the next section).

Remark 9 Note that for the case of continuous PWA systems, the continuous vector field from the state equation (2) given by $f(z, u)=A_{i} z+b_{i}+B_{i} u$ and $f(z(k T), u(k T))=A_{j} z(k T)+b_{j}+B_{j} u(k T)$ is locally Lipschitz in $z$ with Lipschitz constant $L=$ $\max _{i=1, \ldots, M}\left\|A_{i}\right\|$. In this case, following the ideas presented in Khalil (2004), the Gronwall-Bellman inequality applied to the integral of the dynamical equation (2) between $k T$ and $t \leq k T+T$

$$
\begin{gathered}
z(t)=z(k T)+(t-k T) f(z(k T), u(k T))+ \\
\int_{k T}^{t}[f(z(\tau), u(k T))-f(z(k T), u(k T)] d \tau
\end{gathered}
$$

enables us to show that

$$
\begin{array}{r}
\|z(t)-z(k T)\| \leq \frac{1}{L}\left[e^{(t-k T) L}-1\right] . \\
\left\|A_{j} z(k T)+b_{j}+B_{j} u(k T)\right\|, k T \leq t \leq k T+T
\end{array}
$$

When the control input is replaced by its value $u(k T)=$ $K_{j} z(k T)+m_{j}$, it finally yields the bound

$$
\|z(t)-z(k T)\| \leq \frac{1}{L}\left[e^{T L}-1\right]\left[\left\|\bar{A}_{j}\right\|\|z(k T)\|+\left\|\bar{b}_{j}\right\|\right] .
$$

where we have used the fact that $t-k T \leq T$ for $k T \leq$ $t \leq k T+T$ and $\bar{A}_{j}, \bar{b}_{j}$ are defined as before. Following the reasoning leading to (24) a new value for $N_{i j}(k, T)$ 


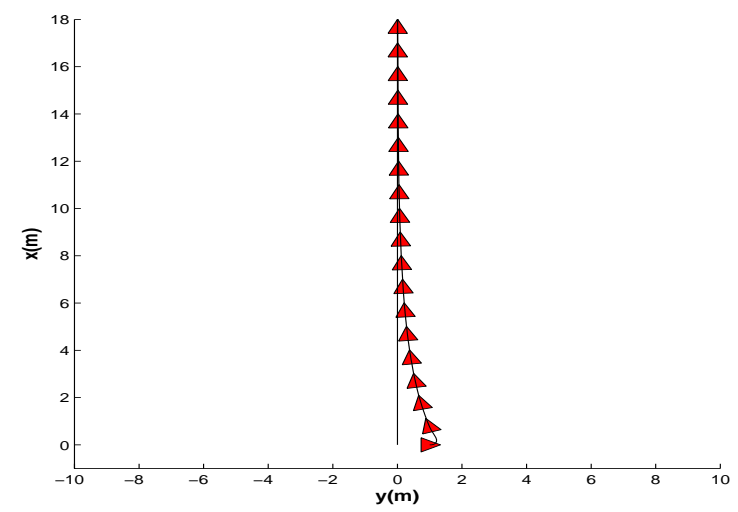

Fig. 1. $x-y$ trajectory with continuous-time controller, $\psi_{0}=\frac{\pi}{2}, r_{0}=0 \mathrm{rad} / \mathrm{s}, y_{0}=1 \mathrm{~m}$

can be found as

$N_{i j}(k, T)=\Delta_{m_{i j}}+\frac{K}{L}\left[e^{T L}-1\right]\left[\left\|\bar{A}_{j}\right\|\|z(k T)\|+\left\|\bar{b}_{j}\right\|\right]$

Note that (24) and_(29) become very similar ( $\bar{A}, \bar{b}$ are replaced by $\left.\left\|\bar{A}_{j}\right\|,\left\|\bar{b}_{j}\right\|\right)$ for very small $T$ if the Euler approximation is used in (24) to approximate $Z(k, T)$ by $\|z(k T)\|$. Note however that (24) is more general and less restrictive than (29) because it is valid even for discontinuous PWA systems that are therefore not locally Lipschitz. The important point to make is that from expression (29) when $T \rightarrow 0, \bar{N}_{i j}(T) \rightarrow 0$ a.e. since $\|z(k T)\|$ is bounded and $z(k T) \rightarrow z(t)$ so that $i, j$ become the same, except on a set of measure zero. This leads to the same result obtained in Corollary 6 when one replaces (24) by (29) for the special case of continuous PWA systems.

\section{Example}

The objective of this example is to design a controller that forces a cart on the $x-y$ plane to follow the straight line $y=0$ with a constant velocity $U_{0}=1 \mathrm{~m} / \mathrm{s}$. It is assumed that a controller has already been designed to maintain a constant forward velocity. The cart's path is then controlled by the torque $u$ about the $z$-axis according to the following dynamics:

$$
\left[\begin{array}{c}
\dot{\psi} \\
\dot{r} \\
\dot{y}
\end{array}\right]=\left[\begin{array}{ccc}
0 & 1 & 0 \\
0 & -\frac{k}{I} & 0 \\
0 & 0 & 0
\end{array}\right]\left[\begin{array}{l}
\psi \\
r \\
y
\end{array}\right]+\left[\begin{array}{c}
0 \\
0 \\
U_{0} \sin (\psi)
\end{array}\right]+\left[\begin{array}{c}
0 \\
\frac{1}{I} \\
0
\end{array}\right] u
$$

where $\psi$ is the heading angle with time derivative $r$, $I=1 \mathrm{Kgm}^{2}$ is the moment of inertia of the cart with respect to the center of mass and $k=0.01 \mathrm{Nms}$ is the damping coefficient. Note that for this example $B_{i}=$ $B_{j}=B, c_{i j}^{T}=\left[\begin{array}{lll}1 & 0 & 0\end{array}\right], c_{i j}^{T} B=0$. The state of the system is $\left(z_{1}, z_{2}, z_{3}\right)=(\psi, r, y)$. Assume the trajectories can start from any possible initial angle in the range

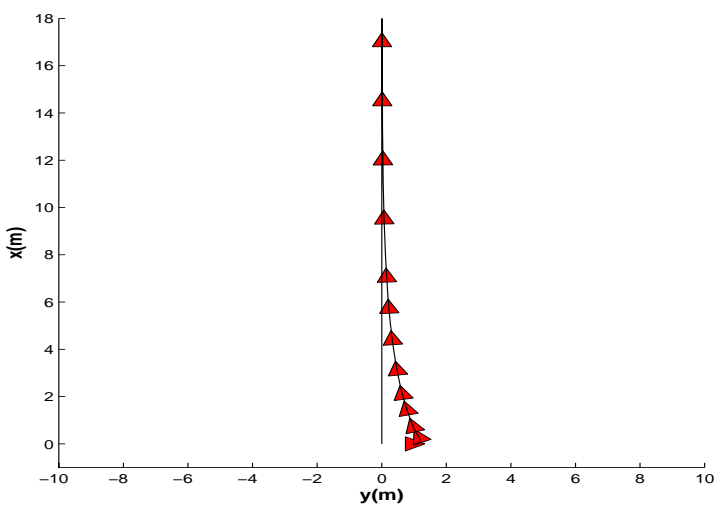

Fig. 2. $x-y$ trajectory for a sampling period of $T=0.05 \mathrm{~s}$

$\psi_{0} \in\left[-\frac{3 \pi}{5}, \frac{3 \pi}{5}\right]$ and any initial distance from the line. The function $\sin (\psi)$ is approximated by a PWA function (see Rodrigues \& How (2001)) yielding

$$
\begin{aligned}
& \mathcal{R}_{1}=\left\{z \in \mathbb{R}^{3} \mid z_{1} \in\left(-\frac{3 \pi}{5},-\frac{\pi}{5}\right)\right\}, \\
& \mathcal{R}_{2}=\left\{z \in \mathbb{R}^{3} \mid z_{1} \in\left(-\frac{\pi}{5},-\frac{\pi}{15}\right)\right\}, \\
& \mathcal{R}_{3}=\left\{z \in \mathbb{R}^{3} \mid z_{1} \in\left(-\frac{\pi}{15}, \frac{\pi}{15}\right)\right\},
\end{aligned}
$$

and $\mathcal{R}_{4}$ is symmetric to $\mathcal{R}_{2}$ and $\mathcal{R}_{5}$ is symmetric to $\mathcal{R}_{1}$, all with respect to the origin. A controller was designed to stabilize the origin (inside region $\mathcal{R}_{3}$ ) yielding

$$
\begin{array}{ll}
K_{1}=[-49.908-9.467-13.926], & m_{1}=2.70 \times 10^{-6} \\
K_{2}=[-48.316-9.330-13.812], & m_{2}=3.75 \times 10^{-7} \\
K_{3}=[-50.148-9.468-13.742] & m_{3}=0.00 \times 10^{0} \\
K_{4}=[-48.316-9.330-13.812] & m_{4}=-m_{2} \times 10^{0} \\
K_{5}=[-49.908-9.468-13.926] & m_{5}=-m_{1} \times 10^{0}
\end{array}
$$

The trajectory in the $x-y$ plane using this controller is shown in figure 1 where it is clear that the controller makes the cart trajectory converge to the desired straight line. For a sampling period of $T=0.05 \mathrm{~s}$ the same controller was emulated in discrete-time between a sampler and a zero-order-hold and the results of the corresponding $x-y$ trajectory are shown in figure 2 . It can be seen that the trajectory still follows approximately the one obtained with the continuous-time controller. When the sampling period is further increased to $T=0.2 s$ the simulation of the $x-y$ trajectory close to the line is zoomed in figure 3 . It is clear that the trajectory converges to a region around the desired straight line, as predicted by the results of this paper. 


\section{Conclusions}

This paper has presented stability results for closed-loop sampled-data PWA systems under state feedback. It was shown that the emulation of a state feedback controller designed in continuous-time to exponentially stabilize the system to a target point would still exponentially stabilize the system to a region around the target point. The size of this region was related to the sampling period. It was shown that when the sampling period converges to zero the exponential stability results for the closedloop continuous-time system are recovered.

\section{Acknowledgements}

The author would like to thank the Associate Editor and the anonymous reviewers for the detailed and interesting suggestions, especially regarding the proof of the Corollary, that greatly improved the quality of the paper. The author would like to also thank Daniel Liberzon and João Hespanha for interesting discussion on this topic. Finally, the author would like to acknowledge the Natural Sciences and Engineering Research Council of Canada (NSERC) for partially funding this research.

Luis Rodrigues earned his "licenciatura" and his M. Sc. degrees in Electrical Engineering and Computers from IST, Technical University of Lisbon. He obtained his Ph. D. in Aeronautics and Astronautics from Stanford University in 2002. He worked as a consultant in speech modeling and recognition for Eliza Corporation in USA and as a project manager for Ydreams in Portugal before joining Concordia as an Assistant Professor in 2003. His research interests lie in the areas of switched, hybrid and optimal control with applications to aerospace, automotive, manufacturing and biological systems (such as the vocal tract).

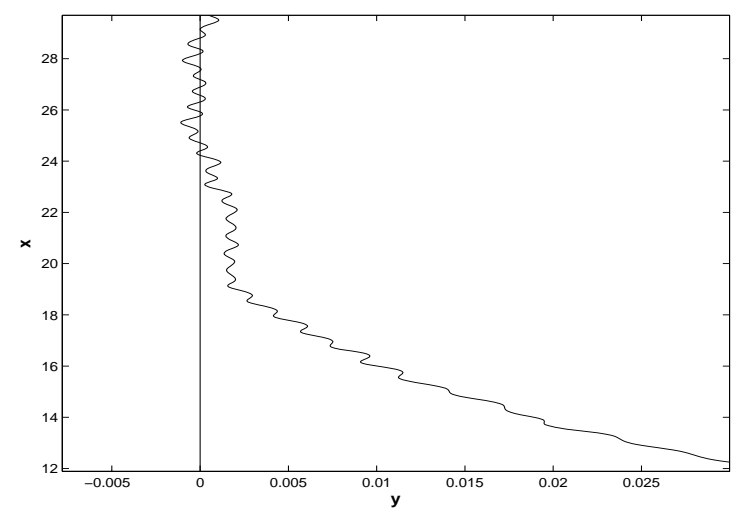

Fig. 3. $x-y$ trajectory zoomed for a sampling period of $T=0.2 s$

\section{References}

S. Azuma \& J. Imura (2004). 'Probabilistic Controllability Analysis of Sampled-Data/Discrete-Time PiecewiseAffine Systems'. In Proc. American Control Conference, pp. 2528-2533.
T. Chen \& B. Francis (1995). Optimal Sampled Data Control Systems. Springer-Verlag.

A. M. G. Zhai, H. Lin \& K. Yasuda (2004). 'Stability Analysis for Switched Systems with Continuous-Time and DiscreteTime Subsystems'. In Proc. American Control Conference, pp. 4555-4560.

A. Hassibi \& S. P. Boyd (1998). 'Quadratic stabilization and control of piecewise-linear systems'. In Proc. American Control Conference, pp. 3659-3664.

J. Imura (2003a). 'Optimal Continuous-Time Control of Sampled-Data Piecewise Affine Systems'. In Proc. American Control Conference, pp. 5317-5322.

J. Imura (2003b). 'Optimal control of sampled-data piecewise affine systems and its application to CPU processing control'. In Proc. of the 42nd IEEE Conf. on Decision and Control, pp. 161-166.

M. Johansson (2003). Piecewise Linear Control Systems. Springer-Verlag, Berlin Heidelberg.

M. Johansson \& A. Rantzer (2000). 'Piecewise linear quadratic optimal control'. IEEE Transactions on Automatic Control 45:629-637.

H. Khalil (2004). 'Output Feedback Sampled-Data Stabilization of Nonlinear Systems'. In Proc. American Control Conference, pp. 2397-2402.

D. Nesic \& D. S. Laila (2002). 'A Note on Input-to-State Stabilization for Nonlinear Sampled-Data Systems'. IEEE Transactions on Automatic Control 47:1153-1158.

D. Nesic \& A. R. Teel (2004). 'A framework for stabilization of nonlinear sampled-data systems based on their approximate discrete-time models'. IEEE Transactions on Automatic Control 49:1153-1158.

L. Rodrigues \& S. P. Boyd (2005). 'Piecewise-affine state feedback for piecewise-affine slab systems using convex optimization'. Systems and Control Letters 54:835-853.

L. Rodrigues \& J. How (2001). 'Automated control design for a piecewise-affine approximation of a class of nonlinear systems'. In Proc. American Control Conference, pp. 3189-3194.

L. Rodrigues \& J. How (2003). 'Observer-based control of piecewise-affine systems'. International Journal of Control 76:459-477.

B. Samadi \& L. Rodrigues (2007). 'Stability Analysis for Filippov Solutions of Piecewise Affine Systems: A Sum of Squares Approach'. In Proc. American Control Conference.

Z. Sun (2004). 'Canonical Froms of Switched Linear Control Systems'. In Proc. American Control Conference, pp. $5182-5187$.

Z. Sun \& S. S. Ge (2002). 'Sampling and control of switched linear systems'. In Proc. of the 41st IEEE Conference on Decision and Control, pp. 4413-4418. 